\title{
Development of an In Vitro Release Test (IVRT) for a Vaginal Microbicide Gel
}

\author{
Ryan R. Klein ${ }^{1}$, Jason Q.Tao², Susan Wilder², \\ Kris Burchett ${ }^{1}$, Quyen Bui ${ }^{1}$, and Kailas D. Thakker ${ }^{1, *}$ \\ 'Analytical Solutions, Inc., Durham, NC 27713 \\ 2International Partnership for Microbicides, Silver Spring, MD 20910
}

e-mail:Kailas.Thakker@asi-rtp.com

\begin{abstract}
Sensitive and reproducible methods to assess in vitro release rates from semisolid products can provide significant value during drug development. The purpose of the present study was to develop and validate an in vitro release test (IVRT) for a topical gel formulation of an HIV microbicide. The method was developed using a vertical diffusion cell system, commercially available synthetic membranes, and HPLC with UV detection. The IVRT method was robust, reproducible, and sensitive to some of the formulation parameters evaluated. The method was applied to evaluate release rates of the active pharmaceutical ingredient from formulations during stability studies.
\end{abstract}

\section{INTRODUCTION}

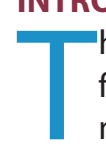

he purpose of a performance test for a dosage form is to predict and monitor the consistency in manufacturing of that dosage form. For topical dosage forms such as gels, creams, and ointments, in vitro release rate testing (IVRT) using the vertical diffusion cell apparatus, or "Franz cell," has become the most well-accepted performance test for semisolid products (1-5).

IVRT can be a useful tool during the development of a topical dosage form. Monitoring the release of a drug from its dosage form during clinical trials can be critical in understanding the efficacy of the formulation in the clinical setting and, in limited cases, has been successful in establishing an in vitro-in vivo correlation for topical products (6). IVRT can be the single test that allows evaluation of drug release from its dosage form following changes to physicochemical properties such as viscosity and particle size, changes in excipients, and changes in manufacturing scale and site. The FDA SUPAC-SS guidance (7) requires IVRT studies following many of these changes to guarantee dosage form performance; thus, IVRT is fast becoming the cornerstone of performance testing for semisolid products.

In the present study, we have developed an in vitro release test for a vaginal gel formulation of a non-nucleoside reverse transcriptase inhibitor that is being developed as a vaginal HIV microbicide. IVRT methods should be reproducible and sensitive to small changes in physicochemical properties of the dosage form. The developed test was robust, precise, and discriminatory, and it served well as a performance test for two lead clinical formulations of the active pharmaceutical ingredient (API) during long-term stability studies.

*Corresponding author.

\section{MATERIALS AND METHODS \\ IVRT Analysis}

Typically, six Franz diffusion cells were used for each experiment. Approximately $300-400 \mathrm{mg}$ of the gel samples were applied in the Teflon wafer of the donor chamber and completely occluded by covering the dosage form with an opaque glass disk. The receiving medium was 40:60 ethanol/pH 4 phosphate buffer. A receiving medium with $40 \%$ ethanol was selected because of the low aqueous solubility of the API. Nylon membrane was selected as the barrier. The temperature of the diffusion cells was maintained at $37^{\circ} \mathrm{C}$, which is typical for vaginal applications. At $0.5,1,2,4$, and $6 \mathrm{~h}, 200 \mu \mathrm{L}$ of sample was withdrawn and replenished with fresh, prewarmed media. API concentration in the IVRT samples was determined using the HPLC method described below. The cumulative amount of API released was calculated and plotted against the square root of time to determine the release rate in each diffusion cell.

\section{HPLC Analysis}

API concentration in the IVRT samples was determined by reversed-phase HPLC with UV detection using a Shimadzu liquid chromatography system. Separation was performed on a Thermo Hypersil BDS C18 column, $10 \mathrm{~cm} \times 4.6 \mathrm{~mm}$ i.d., $5 \mu \mathrm{m}$, maintained at $30^{\circ} \mathrm{C}$. Mobile phase $A$ was $0.5 \%$ ammonium acetate buffer, $\mathrm{pH} 2.3$. Mobile phase $B$ was acetonitrile. A thirteen-minute gradient (Table 1) was used to elute the API with a flow rate of $1.0 \mathrm{~mL} / \mathrm{min}$ and UV detection at $286 \mathrm{~nm}$. Injection volume was $10 \mu \mathrm{L}$. Samples were kept at ambient temperature during analysis.

\section{RESULTS AND DISCUSSION}

Release Rate Dependence on Synthetic Membrane

Three synthetic membranes, nylon, silastic, and cellulose, were selected to study the in vitro release of the gel formulations. Membrane-binding studies were performed to determine whether the API binds to a specific 


\begin{tabular}{lcc}
\hline \multicolumn{2}{l}{ Table 1. HPLC Gradient Profile } \\
\hline Time (min) & $\%$ A & $\%$ B \\
\hline 0 & 70 & 30 \\
\hline 7 & 30 & 70 \\
\hline 7.5 & 0 & 100 \\
\hline 11 & 70 & 30 \\
\hline 13 & 70 & 30 \\
\hline
\end{tabular}

membrane. A solution of the API in receiving media was filtered through each membrane, and the filtrate was collected in 1-mL aliquots; each filtrate was compared to an unfiltered sample (Table 2). Filtration through both nylon and silastic membranes gave complete API recovery, but incomplete recovery through a cellulose membrane. Cellulose was therefore excluded from further evaluation.

Release rates of the API were then determined using both nylon and silastic membranes (Table 2). With silastic membranes, API release rates were very low; very little was found in receiver chambers for up to six hours. Release rates were much higher and more reproducible when the nylon membrane was used. On the basis of these data, nylon was selected as the membrane for further evaluation.

\section{Release Rate Dependence on Air Exposure During Preparation}

The gel samples were generally occluded from air exposure during release rate testing; however, some air exposure was inevitable during the setup of the experiment. The release of the API could have been affected if exposure to air was excessive. Experiments were designed with exposure times of up to one hour, which is significantly longer than the average setup time in a typical experiment ( $2.5 \mathrm{~min})$, and release rates were determined. Figure 1 illustrates that exposure to air for up to one hour longer than the average setup time had no effect on the release rate of the API.

\section{Consistency of Release Rate Measurement over Time}

Release rate values were determined for different batches of one formulation over a period of 812 days

Table 2. Membrane Binding and Average Release Rates for Gel Formulation (0.025\% w/w API) Across Three Types of Synthetic Membranes $(n=3)$

\begin{tabular}{lcccc}
\hline Membrane & $\begin{array}{c}\text { Membrane } \\
\text { Binding } \\
(\% \text { Recovery) }\end{array}$ & $\begin{array}{c}\text { API Release } \\
\text { Rate } \\
\left(\boldsymbol{\mu g} / \mathbf{c m}^{\mathbf{2}} / \mathbf{h}^{\mathbf{0 . 5})}\right.\end{array}$ & $\begin{array}{c}\text { Standard } \\
\text { Deviation }\end{array}$ & \% RSD \\
\hline Nylon & $97.6 \pm 3.0$ & 5.52 & 0.14 & 2.6 \\
\hline Silastic & $99.2 \pm 1.6$ & 0.23 & 0.12 & 53 \\
\hline Cellulose & $92.6 \pm 4.1$ & Not tested & Not tested & Not tested \\
\hline
\end{tabular}

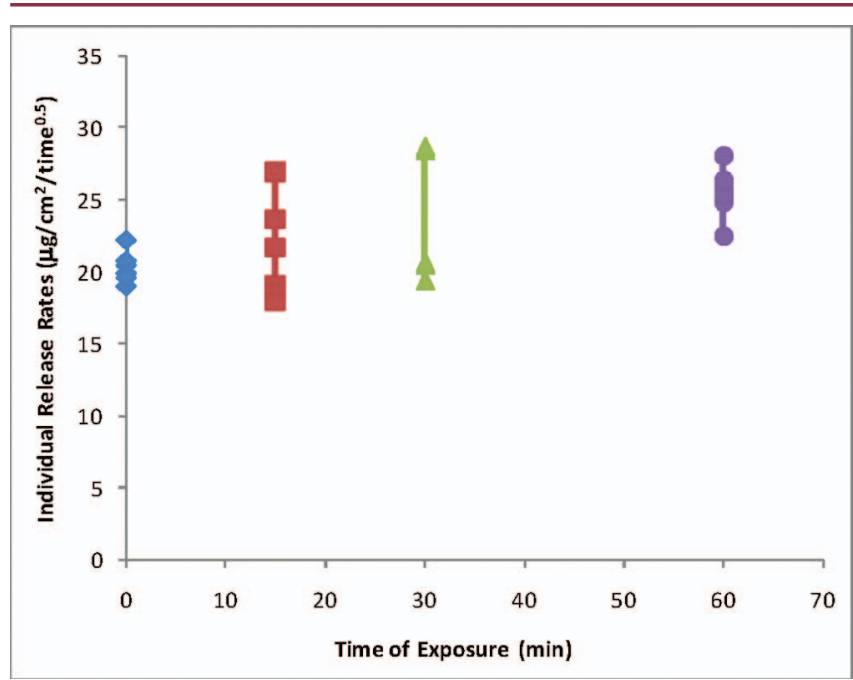

Figure 1. Individual release rates of API following different exposure time to air during set up $(n=6)$.

(twenty-seven months) to assess the reproducibility of the IVRT method. Individual release rates for each of thirteen experiments are presented as a function of time in Figure 2 ; the release rates obtained over this period were within experimental variability typically encountered during IVRT studies. The average release rate was $21.0 \pm 2.87 \mu \mathrm{g} / \mathrm{cm}^{2} /$ $h^{0.5}$ with a coefficient of variation of $13.6 \%(n=90)$. The low coefficient of variation found over this extended time period implies both robust and reproducible manufacturing methods and a robust and reproducible IVRT method. Importantly, these data demonstrate that in vitro release testing is capable of providing an accurate measure of the stability and performance of batches over prolonged periods.

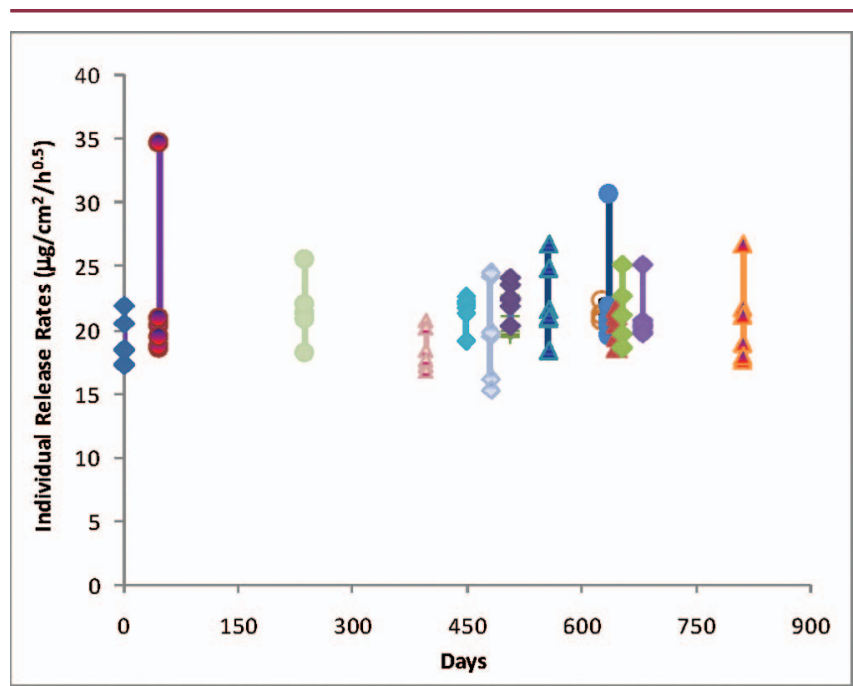

Figure 2. Individual release rates of API released from different batches of the same formulation over a period of twenty-seven months $(n=6)$. Different colors represent different batches of APl; each batch was evaluated on a different day. 


\begin{tabular}{|c|c|c|c|c|}
\hline API $(\% w / w)$ & Formulation & $\begin{array}{l}\text { API Release Rate } \\
\left(\mu \mathrm{g} / \mathrm{cm}^{2} / \mathrm{h}^{0.5}\right)\end{array}$ & $\begin{array}{l}\text { Standard } \\
\text { Deviation }\end{array}$ & \%RSD \\
\hline 0.01 & API, 2\% vitamin E TPGS, 28\% propylene glycol, modified polymers, pH6 & 2.90 & 0.64 & 22 \\
\hline 0.01 & API, 2\% Tween 20, 28\% propylene glycol, modified polymers, pH6 & 5.63 & 0.36 & 6.4 \\
\hline 0.01 & API, $2 \%$ Cremophor, $28 \%$ propylene glycol, modified polymers, $\mathrm{pH} 6$ & 5.86 & 0.93 & 16 \\
\hline 0.01 & API, 40\% propylene glycol, modified polymers, $\mathrm{pH} 7$ & 6.14 & 1.2 & 19 \\
\hline 0.025 & API, $2 \%$ Cremophor, $28 \%$ propylene glycol, modified polymers, $\mathrm{pH} 6$ & 11.7 & 1.1 & 9.3 \\
\hline 0.025 & API, 2\% vitamin E TPGS, 28\% propylene glycol, modified polymers, pH6 & 11.9 & 1.3 & 11 \\
\hline 0.025 & API, $2 \%$ Tween $20,28 \%$ propylene glycol, modified polymers, $\mathrm{pH} 6$ & 13.9 & 2.0 & 15 \\
\hline 0.025 & API, vitamin E TPGS, 5\% propylene glycol, modified polymers, $\mathrm{pH} 6$ & 14.7 & 2.3 & 16 \\
\hline 0.025 & $\mathrm{API}, 4 \%$ ethanol, modified polymers, $\mathrm{pH} 6$ & 15.4 & 2.0 & 13 \\
\hline
\end{tabular}

\section{Release Rate Dependence on Formulation Composition}

Figure 3 and Table 3 show the release profiles and release rates of nine different formulations with varying compositions. Within the same dose group, release rates varied as much as two-fold. Differences within each dose group are likely attributable to the differences in the excipients present in each formulation, thus demonstrating the ability of the IVRT method to discriminate among formulations.

\section{Release Rate Dependence on Dose Strength}

Various dose strengths of two lead formulations of the API gel were evaluated. Figure 4 illustrates the release rate profiles of the two formulations at three dose strengths. For Formulation 1, although the method could discriminate among the tested doses, the relationship between release rate and dose strength was not linear. The highest dose tested was not proportional to the determined release rate, revealing a profile that indicated saturation of the release rate. Limited solubility of the API in the receiving media is an unlikely cause of this saturation because the measured solubility of the API in

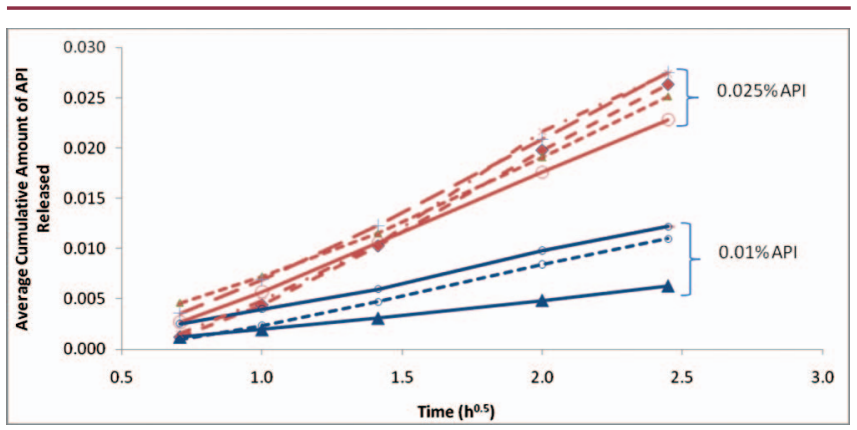

Figure 3. API release profiles from various formulations at two dose strengths $(n=6)$. Red lines with open markers are $0.025 \%$ API gels, while blue lines with filled markers are $0.01 \%$ API gels. Error bars have been omitted for clarity. the receiving media was approximately three times the highest detectable concentration. Furthermore, for Formulation 2, the relationship between release rate and dose strength was linear over the same range of doses; therefore, the saturation of drug release observed with Formulation 1 is likely due to physicochemical properties of the formulation itself, rather than solubility limitations in the receiving media.

\section{Release Rate Dependence on Particle Size}

The effect of API particle size reduction on its release rate was also assessed.The mean particle size of nonmilled API was approximately $144 \mu \mathrm{m}$, while two milling processes yielded reduced mean particle sizes of approximately $15 \mu \mathrm{m}$ and $5 \mu \mathrm{m}$. Figure $5 \mathrm{~A}$ illustrates the release rate profiles of the three formulations containing API of different particle sizes, while Figure $5 B$ presents the individual release rates from each formulation. Although the nonmilled API formulation demonstrated a trend toward reduced release rates, the profiles of all three formulations were within experimental variability. This

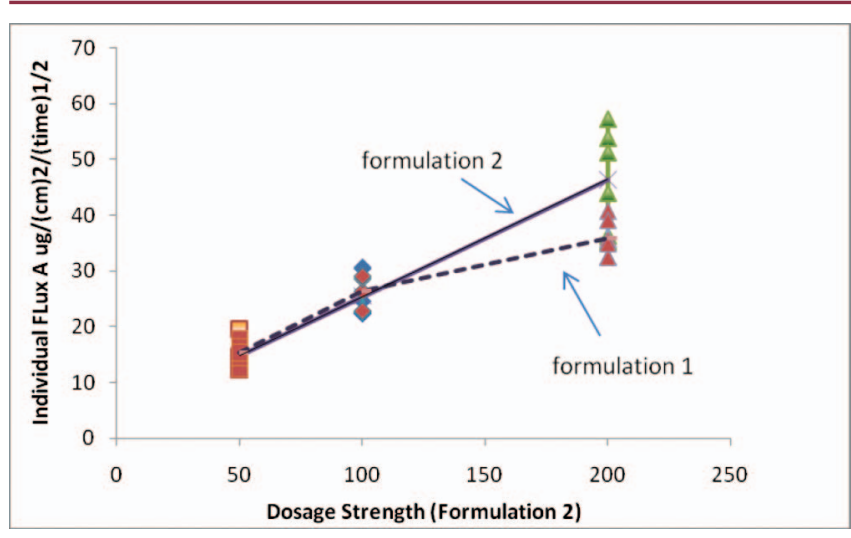

Figure 4. Individual release rates of API from Formulations 1 and 2 at different dose strengths $(n=6)$. 


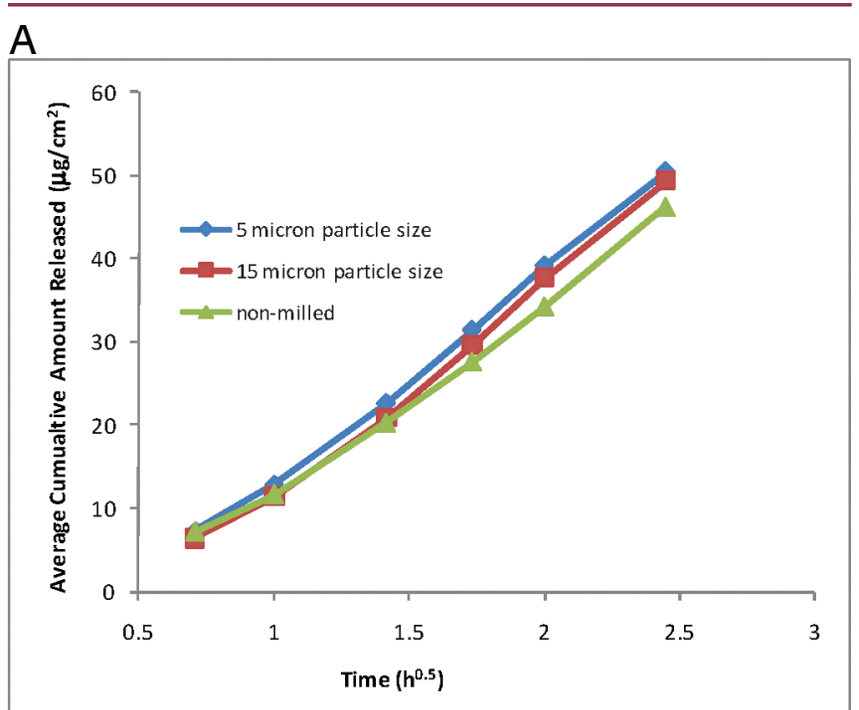

B

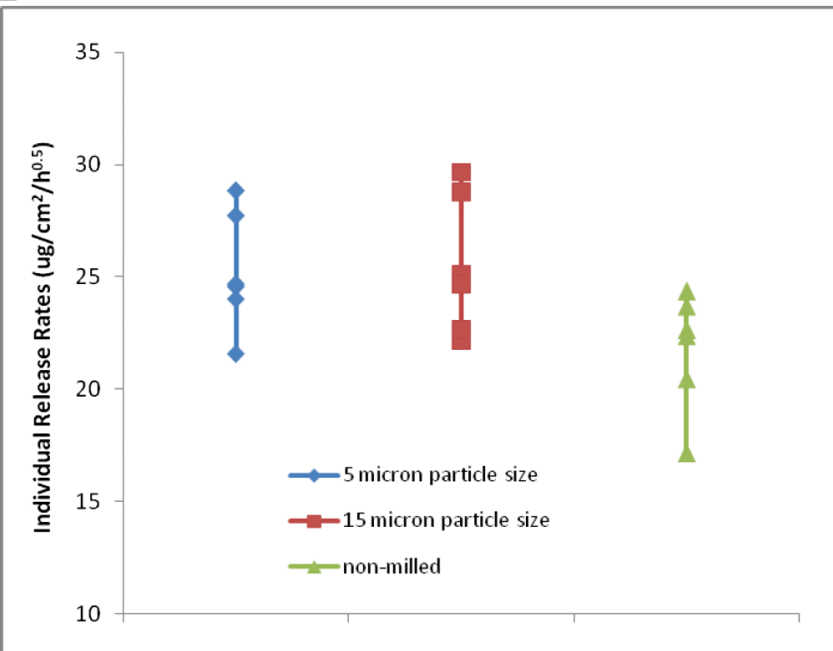

Figure 5. (A) API release profiles from formulations prepared with various particle sizes $(n=6)$. Error bars have been omitted for clarity. (B) Individual release rates of API released from formulations prepared with various particle sizes $(n=6)$.

result implies that (1) the developed IVRT method is not sensitive to changes in API particle size from the tested formulation or (2) the release rate of the API is not affected by the particle size range tested.

\section{Release Rate Dependence on Stress-Induced Viscosity Changes}

Changes in the viscosity of gel formulations lead to altered drug release rates (8). The current method was evaluated for its ability to discriminate stress-induced viscosity changes. The viscosity change was induced by stressing Formulation 3 at $60^{\circ} \mathrm{C}$ for a period of four weeks; the viscosity of the formulation increased from 8.3 to $10.8 \mathrm{kPa}$ under these conditions. Figure 6 illustrates the release profiles of stressed and unstressed formulations. The increase in viscosity led to a decrease in the release rate of the API from $25.6 \pm 2.7$ to $20.6 \pm 0.84 \mu \mathrm{g} / \mathrm{cm}^{2} / \mathrm{h}^{0.5}$.

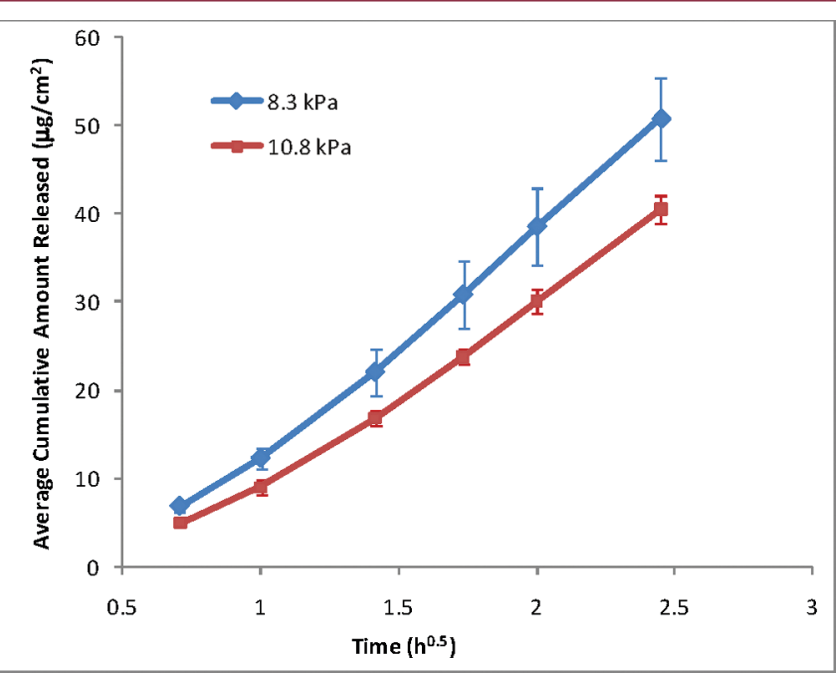

Figure 6. API $(0.025 \% \mathrm{~W} / \mathrm{W})$ release profiles from formulations of different viscosity $(n=6)$.

Under these experimental conditions, the Wilcoxon Rank Sum/Mann-Whitney statistical test was applied; the two release profiles were confirmed to be significantly different.

\section{Application of the IVRT Method to Release Rate Monitoring During Stability Testing}

Evaluation of release rates during stability testing can provide valuable information about the continued performance of the dosage form. The developed IVRT method was utilized to assess the release rate of the API from different batches of two lead gel formulations on stability testing at $30{ }^{\circ} \mathrm{C} / 65 \%$ relative humidity and $40^{\circ} \mathrm{C} / 75 \%$ relative humidity. Previous studies (Figures 2 and 6) had already established both the reproducibility of the IVRT method over a period of twenty-seven months and the potential impact of stress (storage at increased temperature) on semisolid gel formulations of the API.The data presented in Figures $7 \mathrm{~A}$ and $7 \mathrm{~B}$ demonstrate that the release rate of the API from Formulation 2 was not affected by the accelerated stability conditions; in fact, the release rates of the API from both formulations were very consistent over the periods tested. (Release rates from Formulation 1 are not shown.)

\section{CONCLUSION}

In the last decade, in vitro release testing using vertical diffusion cells has demonstrated promise as a tool to assess the release of active pharmaceutical ingredients from semisolid products. Recent data have demonstrated the robust and reproducible nature of such studies (9); however, it is clear that additional evidence is necessary to establish this test as a true performance test for topical dosage forms. In the present study, an in vitro release test was developed for a vaginal microbicide. The IVRT method was utilized to evaluate various parameters of potential gel formulations and to monitor the change in release rate of the API from formulations during stability testing. 


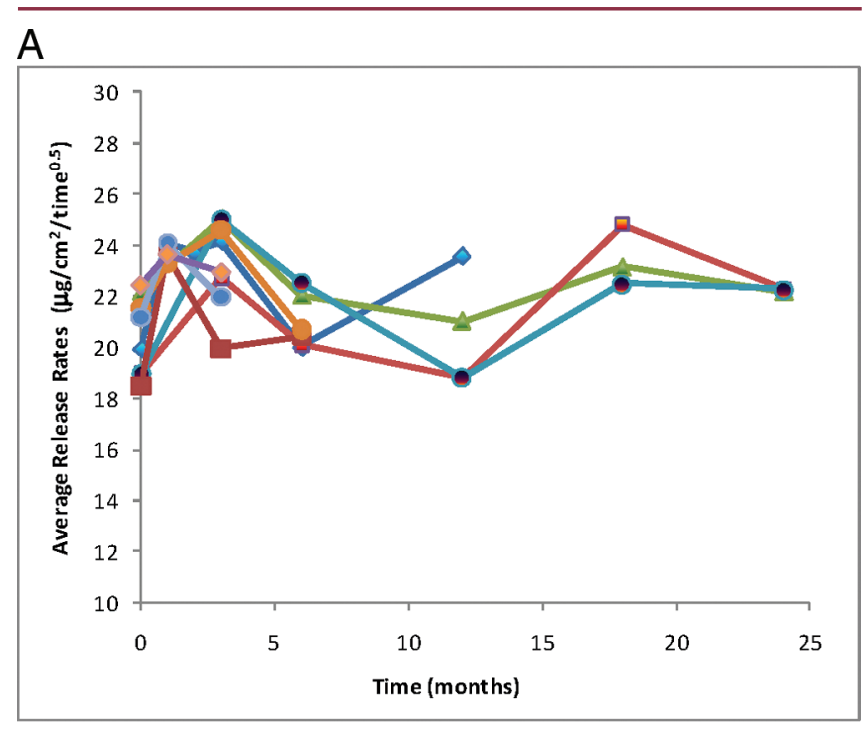

B

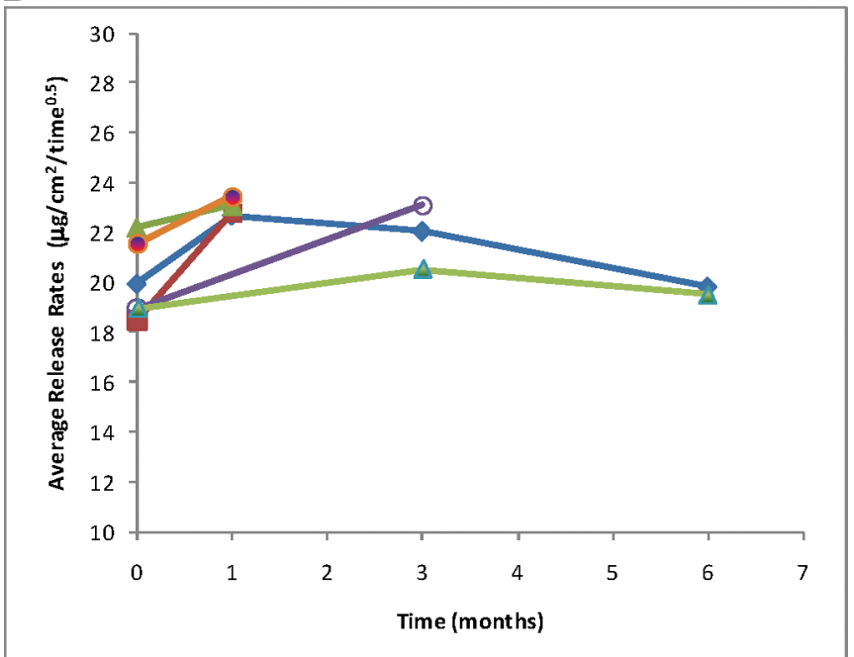

Figure 7. (A) Average release rates of API released from different batches of the same formulation over twenty-four months of storage at $30^{\circ} \mathrm{C} / 65 \% \mathrm{RH}$ $(n=6)$. Different colors represent different batches of formulation. Error bars have been omitted for clarity. (B) Average release rates of API released from different batches of the same formulation over six months of storage at $40^{\circ} \mathrm{C} / 75 \% \mathrm{RH}(n=6)$. Different colors represent different batches of formulation. Error bars have been omitted for clarity.

Of particular importance for a release testing method is its sensitivity to both chemical and physical changes to the formulation such as viscosity, excipient composition, and particle size of the active pharmaceutical ingredient. The in vitro release test developed for the vaginal gel was precise, robust, and reproducible and was discriminatory for some of the evaluated formulation parameters. The method proved to be particularly sensitive to formulation composition and viscosity. A modest increase in viscosity led to a significant decrease in the release rate of the API, while excipient composition changes resulted in as much as a two-fold change in release rate. Importantly, the IVRT method produced comparable release rates for different batches of the same gel formulation over a period of twenty-seven months, suggesting that the method can be reliably used to monitor and gauge consistency and quality in manufacturing of future batches. Finally, the developed IVRT method served well as a performance test for the two lead clinical formulations of the API during long-term stability studies.

\section{ACKNOWLEDGMENTS}

Thanks to Kristina Kenney from Analytical Solutions Inc. and Piotr Bryla from International Partnership for Microbicides for their contributions to the work. This work was funded by the International Partnership for Microbicides.

\section{REFERENCES}

1. Shah, V. P.; Elkins, J.; Hanus, J.; Noorizadeh, C.; Skelly, J. P. In vitro release of hydrocortisone from topical preparations and automated procedure. Pharm. Res. 1991, 8 (1), 55-59.

2. Shah, V. P.; Elkins, J. S.; Williams, R. S. Evaluation of the test system used for in vitro release of drugs for topical dermatological drug products. Pharm. Dev. Technol. 1999, 4 (3), 377-385.

3. Flynn, G. L.; Shah, V. P.; Tenjarla, S. N.; Corbo, M.; DeMagistris, D.; Feldman, T. G.; Franz, T. J.; Miran, D. R.; Pearce, D. M.; Sequeira, J. A.; Swarbrick, J.;Wang, J. C.T.; Yacobi, A.; Zatz, J. L. Assessment of value and applications of in vitro testing of topical dermatological drug products. Pharm. Res. 1999, 16 (9), 1325-1330.

4. Proniuk, S.; Dixon, S. E.; Blanchard, J. Investigation of the utility of an in vitro release test for optimizing semisolid dosage forms. Pharm. Dev. Technol. 2001, 6 (3), 469-476.

5. Shah, V. P.; Elkins, J. S.; Shaw, S.; Hanson, R. In vitro release: Comparative evaluation of vertical diffusion cell system and automated procedure. Pharm. Dev. Technol. 2003, 8 (1), 97-102.

6. Shah, V.P.IV-IVC for topically applied preparations-a critical evaluation. Eur.J. Pharm. Biopharm. 2005, 60, 309-314.

7. Nonsterile Semisolid Dosage Forms, Scale-Up and Postapproval Changes: Chemistry, Manufacturing, and Controls; In Vitro Release Testing and In Vivo Bioequivalence Documentation; Guidance for Industry; U.S. Department of Health and Human Services, Food and Drug Administration, Center for Drug Evaluation and Research (CDER), U.S. Government Printing Office: Washington, DC, 1997.

8. Moreira,T.S.; de Sousa, V.P.; Pierre, M. B. Influence of oleic acid on the rheology and in vitro release of lumiracoxib from poloxamer gels. J. Pharm. Pharm. Sci. 2010, 13 (2), 286-302.

9. Hauck, W.W.; Shah, V.P.; Shaw, S.W; Ueda, C.T. Reliability and reproducibility of vertical diffusion cells for determining release rates form semisolid dosage forms. Pharm. Res. 2007, 24 (11), 2018-2024. 